\title{
Research on the New Full View Detection Equipment of Bridge Superstructure
}

\author{
Jinbo Song \\ Jiangxi Vocational and Technical College of Communication, china \\ jxjtxylqx@126.com
}

\begin{abstract}
Keywords: Bridge detection; full angle detection; CCD camera; bridge management system; robot. Abstract. New perspective of the upper structure of the bridge detection device comprises a detection device body, detection support, crawling mechanism, gas spring, motor, image acquisition device, the buckle plate, the chassis and the control device, the detection device is installed on the cable body, body detection device comprises a detection bracket, crawling mechanism, motor, image acquisition detection device, control device, supporting frame set in the cable outside the support frame is installed on the inner wall detection creeping mechanism, crawling mechanism is connected with a motor side; the detection bracket is installed at the bottom of the chassis, the chassis is provided with a control device, photoelectric encoder and communication device; the detection bracket is installed on the upper part of the image acquisition device the image acquisition device, and control device for electrical connection. The utility model can judge the external damage of the cable, which ensures the accuracy and safety of the upper air detection, and has the advantages of light weight, easy installation and high intelligent degree, and can better maintain the safety of the bridge.
\end{abstract}

\section{Introduction}

Bridge construction is one of the important national infrastructure, the rapid development of bridge construction, the huge capital investment, of prominent role in the economy and society, making people on the bridge safety, durability, more and more attention. Oblique pulling bridge, suspension bridge, concrete filled steel tube arch bridge model of cable supporting system bridge, due to the obvious characteristic of the spanning capacity, beautiful shape, seismic ability strong, more and more appear in rivers. These bridges in the operation of the process, the cable structure is safe or not, directly related to the bridge structure and the people's lives and property safety. The bridge is faced with a large number of bridges has entered the aging stage, due to the lack of modern bridge detection equipment and information management maintenance methods, resulting in some bridges have been hidden, cause the bridge crash events occur from time to time. These major security incidents to the country's economic property and the people's lives and safety has brought immeasurable and irreparable loss, but also exposed the existing bridges in the detection and safety assessment methods have many deficiencies.

Suspender cable is a cable stayed bridge and suspension bridge model bridge an important component and an important part of, its cost is very expensive, about one-third of the cost of the bridges in about, due to the long-term exposure to the atmosphere by wind blowing, insolation, rain and pollution of the environment of the erosion, the surface of the polyethylene sheath will produce different degrees of hardening and cracking phenomenon, and then to the sheath of the wire bundle bring rust, broken wire and other serious problems, its corrosion damage is most cable stayed bridge and suspension bridge accidents important reasons. Therefore, periodic inspection and maintenance of cable stayed bridge is needed.

\section{Existing Detection Methods and Research Status at Home and Abroad}

Existing Detection Methods. For detection of appearance defects of cable (boom), currently the industry mainly uses is manual detection. Using this detection method is hoisting carriage equipped with artificial from the cable to the bottom of the cable to the top of the cable detection, this detection method is not only low efficiency, high cost, and has a big risk. The hoisting trolley weight reached 
hundreds of kilograms, the cable itself is a kind of damage and detection efficiency and accuracy of the human eye detection method for low, and the high risk, often leading to the following two situations: the first is cable has significant quality problem and not detected, causing the bridge to collapse, causing major traffic accidents; the second is conservative estimation of the cable quality, lead to unnecessary cable replacement, resulting in waste of a large amount of manpower, material and financial resources. For existing testing, often used to detect vehicles and large lifting equipment, the need for fully closed or semi closed traffic, a serious obstacle to the operation of traffic. At present, the cost of traditional bridge inspection car is huge. Work requires a semi closed traffic, to see a bridge to spend a few days a few days, not suitable for the needs of modern bridge maintenance management. Due to bridge subjected to the specific environment and objective condition limit, existing approaches difficult on the bridge cable (boom) all were detected, can only choose a representative to inspect the parts, thus reducing the we use grasp the extent of the status of the bridge, and obsolete equipment, backward nature cannot reflect the bridge all the status quo, serious impact on the assessment of the quality of the bridge. At present, the domestic R \&amp; D of bridge cable inspection robot because of the weight is too heavy, intelligent degree is not high, the use is not convenient and so on, and not in the industry. Therefore, it is necessary to design a fast, efficient and accurate detection equipment, in order to shorten the time of bridge detection, improve the accuracy of detection, and reduce the impact on the normal traffic.

At present, it is difficult to be applied in bridge pipe support and detection, and it is more difficult to meet the requirements of routine testing of bridge, as shown in Fig. 1- Fig. 4.

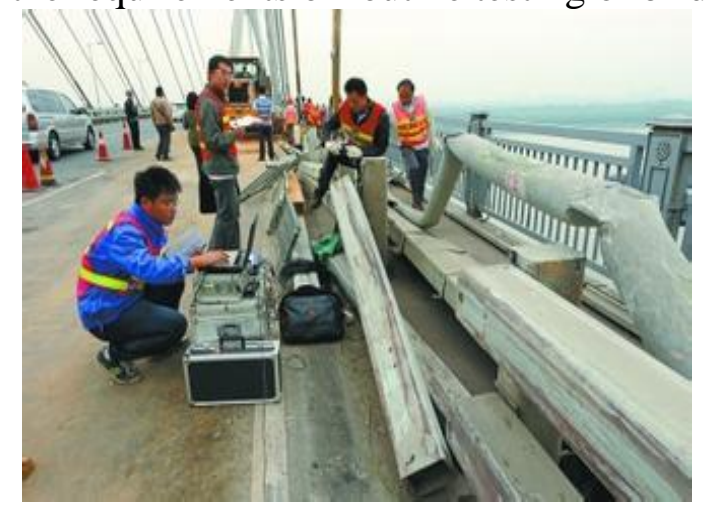

Fig. 1 hydraulic crane tower test

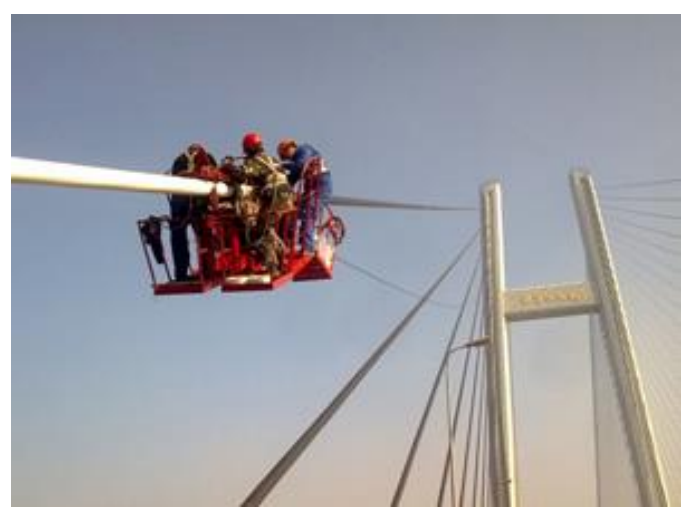

Fig. 3 staff in basket testing

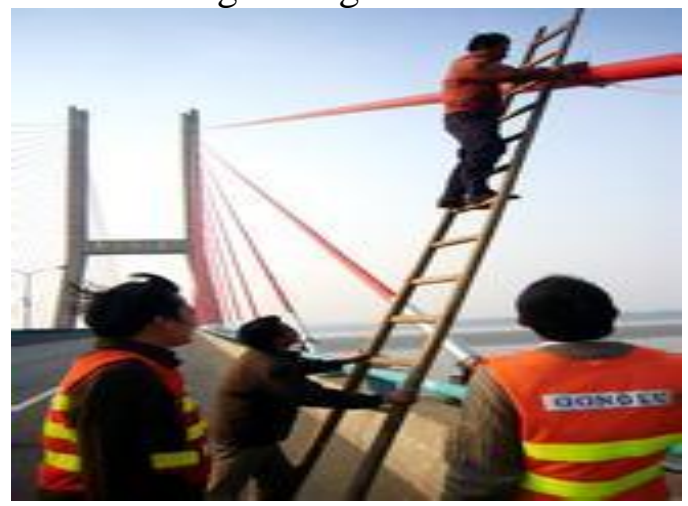

Fig. 2 the detection of artificial climbing rope

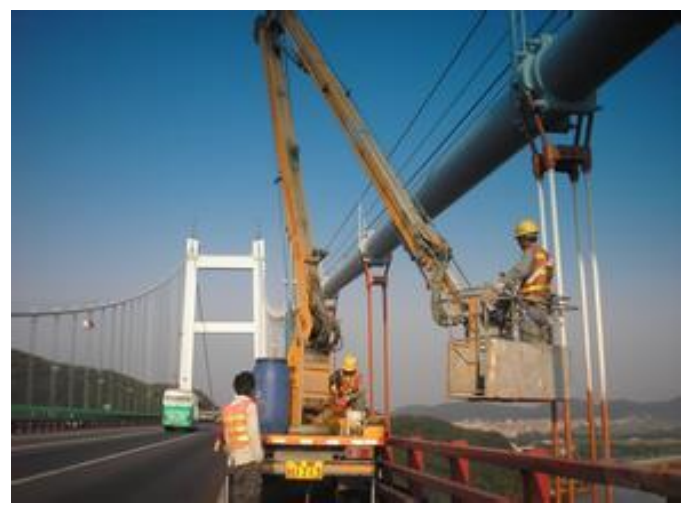

Fig. 4 large bridge detection vehicle cable detection

Current Research Situation of Domestic and International Testing Equipment. Domestic and foreign scholars have been proposed by cable climbing automatic detection device or a robot for bridge cables were detected. This method has good automatic and intelligent and can give cable-stayed bridge cable detection and maintenance operations in addition to manual detection method of an important means, certain extent can get rid of the risk brought by manual inspection.

The United States, Japan, Canada and other developed countries, due to the needs of the development of transportation industry, have carried out a to pavement damage to computer image 
real-time acquisition device (hardware) and pavement image automatic processing system (software) for the main content of the research and development work, and in the early 1990s and continued to publish the results of their research. Development of a detection system mainly: American ARIATM system, PCES system, Japan's Komatsu system, Switzerland CREHOS system, Canada the wisecrax system etc., the system can automatic real-time of crack identification, generation include crack position, geometry such as length, width, area and other content, crack pattern, and the longitudinal cracks, lateral cracks, block cracks and cracks types of cracks are classified, and at the same time automatically establish the image database.

At home and abroad, the cable (boom) detection equipment has a huge body, complex structure, high cost of use and maintenance. Lack of necessary bridge cable (boom) image recognition and defect evaluation system, and information management tools, and not in the bridge detection and management of the maintenance of a good use. Therefore, it is necessary to develop a convenient, low-cost cable rapid detection equipment, the use of modern information technology, for bridge cables (boom) for rapid detection and evaluation and the formation of bridge health and safety archives to adapt to the domestic and foreign new bridge maintenance and need to deal with.

\section{Bridge Superstructure New Full View Detection Equipment}

Equipment Overall Design Ideas. This set of equipment mechanical structure, electrical and mechanical equipment, electronic communications, wireless transmission, automatic control, cable structure evaluation and repair and other functions, high technology content, strong innovation; the all wheel drive, climbing back, automatic navigation, positioning, to ensure the accuracy and safety of high altitude detection; equipped with high resolution digital camera, cable detection system, evaluation of external cable defects, according to the needs of future, can be equipped with automatic cable winding system, cable appearance defect repair system, can be detected, cable structure assessment and maintenance of integrated security maintenance platform, has the advantages of light weight, easy installation, high intelligence characteristics. To better safeguard the safety of bridge. Can provide strong technical support for the structural safety assessment of the cable system.

Equipment Working Process. Bridge superstructure full angle detecting device in the working process, the crawler wheel can drive detection device body climb, decreased or hovering, through the buckle and a spring rod can be adjusted to adapt to the different thickness of the cable, a CCD image acquisition equipment to the cable around the complete graph image acquisition to control device, synthesis, processing and defect recognition. Control device built-in DSP chip, the data processing and storage, and data through the communication device to send to the operator's mobile terminal, easy to understand and operate in real time.

The equipment includes a bracing frame, crawling mechanism, gas spring, a motor, a image acquisition device, card buckle plate, a chassis and a control device for detecting device body detection, the detecting device is installed on the cable. Detection device body comprises a detecting support frame, crawling mechanism, motor, image acquisition device, a control device to detect support frame is sheathed on the outside of the cable, detection support frame wall is installed on the crawling mechanism, crawling mechanism side is connected with a motor; the detection the bottom of the support frame is installed chassis, the chassis is provided with a plurality of air spring, the chassis is also provided with a control device, photoelectric encoder and communication device; the support frame at the upper part of the installation of a plurality of image acquisition device and image acquisition device and control device is electrically connected. The crawling mechanism comprises three crawler wheel mechanisms, and the crawler wheel mechanism is uniformly distributed along the circumference of the cable center, and the crawling mechanism is hinged with the detection support frame through a connecting rod. Detection side surface of the supporting frame is provided with a sliding block, sliding block detection along the support frame on the side of the slide, crawler wheel mechanism is connected with a spring rod, bar spring and the end part of the sliding block hinged and sliding block is connected with the top of the air spring, the number of gas spring for three. Image acquisition device for $\mathrm{CCD}$ image acquisition equipment, the number of image acquisition 
device for the four, the image acquisition device installed on the same plane evenly distributed. The communication device is a wireless communication or Bluetooth communication device, a communication device and a photoelectric encoder are connected with the control device. The bottom of the base plate is provided with a clamping buckle plate.

\section{Summary}

China currently has a variety of types of highway bridges 904 thousand and 600, according to the relevant standards need to conduct regular testing of these bridges, in order to assess the health status and operational capacity of these bridges. If the works maintenance warning management of existing bridges, each bridge every year can save test cost an average of 10 million yuan, calculated to 5 years for a bridge safety management cycle, every can save conventional bridge testing costs about 297.3 billion yuan, and each year to provide about nearly 2 million jobs and 100 billion yuan of tax and economic benefits significantly. Various types of bridges are gradually shifting from the construction stage to the maintenance and maintenance to ensure the safety of the highway and bridge structure. A large number of bridge structures, especially the urban road and bridge, have been damaged and damaged due to the influence of service life, traffic load and load level, natural factors and overload factors. Especially after the nineties of the last century built in large numbers of urban bridges, many of which have appeared in the crack and deflection of the mid span and other diseases that pose a threat to the safe operation of the bridge, the urgent need of the bridge of the state of real-time detection and objective evaluation. In the current urban road maintenance and management, mainly to artificial periodic inspection and evaluation of main, because of the lack of professional equipment and relevant experience are often difficult to detect disease, also cannot achieve timely and effective maintenance, and this essay expounds the application equipment not only can realize timely detection of disease and take effective measures to deal with the will from a macroscopic point of view of the whole urban road network to achieve effective monitoring and management, is of great practical significance and social benefits.

\section{Acknowledgements}

This work reported here was supported by the Jiangxi Provincial Department of Education Science and Technology Fund Project (GJJ151425 and GJJ151419). Any opinions, findings and conclusions or recommendations expressed in this paper are those of the authors and do not necessarily reflect those of the sponsor.

\section{References}

[1] Peng Liming, Chen Lei, Gao Zhiyong. A design and research of the robot for the detection of the cable with adjustable cable force, 2012,, 28 (3): 21-23.

[2] Gao Gao, Peng Liming. The design of the robot body for the detection of the cable in the Hangzhou Bay sea crossing bridge [J]. Shanxi architecture, 2012, 38 (22): 174-175.

[3] Zhang Ni. Germany Elbe bridge detection system [J]. world bridge, 2015 (5): 94-95.

[4] accounting fine, Zhou Yu, Ye Qingwei, et al. The detection method of cable force of cable-stayed bridge based on ERA algorithm [J]. Journal of electronic measurement and instrument, 2010, 24 (8): 743-747.

[5] Peng Liming, Gao Zhiyong, Chen Lei, et al. Design and analysis of a multi roller frame type climbing robot $[\mathrm{J}]$. mechanical science and technology, 2014 (4). 